\title{
EL POTENCIAL DE LOS PROYECTOS DE APRENDIZAJE TUTORADO EN LA FORMACIÓN INICIAL DEL PROFESORADO
}

The potential of tutored learning projects in initial teacher training

\begin{abstract}
Este estudio se ha llevado a cabo dentro del proyecto de I+D+i: "La competencias docentes en la formación inicial del profesorado de educación física”. Convocatoria de noviembre de 2013 del Programa Estatal de Investigación, Desarrollo e Innovación Orientada a los Retos de la Sociedad, en el marco del Plan Estatal de Investigación Científica y Técnica y de Innovación 2013-2016. Referencia: EDU 2013-42024-R. Duración: 3 años (2014-2016).
\end{abstract}

\section{Juan Carlos Manrique Arribas}

Facultad de Educación de Segovia, Universidad de Valladolid, España. Teléfono: +34921112206. Correo electrónico: manrique@mpc.uva.es

\section{Resumen}

La autonomía no se alcanza con el simple “dejar hacer” cuando se encarga un trabajo en grupo o un proyecto. Para ayudar a desarrollar la autonomía en los alumnos es fundamental enfrentarles a un problema auténtico, en el que tengan que tomar decisiones y contrastarlas en la práctica. Pero también es fundamental acompañarles en el recorrido y ayudarles a que tomen conciencia de los errores y aciertos que realizan. También es conveniente que aprendan a programar sus unidades y sesiones y que adviertan las posibles diferencias entre aplicar sesiones prácticas con sus compañeros y con alumnos reales en un centro de Primaria. Por tanto, la realización de Proyectos de Aprendizaje Tutorado (PAT) es una adecuada estrategia metodológica para alcanzar las competencias profesionales que debe adquirir el alumnado del Grado en Educación Primaria durante su proceso formativo.

Palabras clave: proyectos de aprendizaje tutorados; evaluación formativa; competencias

\begin{abstract}
Autonomy is not reached with the simple "laissez-faire" when students have to do group work or a project. In order to help students develop autonomy, it is essential to confront


them with a real problem, in which they have to make decisions and examine them in light of their practice. It is also essential to accompany them throughout the process and help them to become aware of their own mistakes and successes, and help them to become aware of their own mistakes and successes. It is also convenient that students learn to plan their units and sessions, and that they notice potential differences between applying practical sessions with peers and with real students in a Primary School. Therefore, carrying out "PAT", (Tutored Learning Projects), constitutes a proper methodological strategy to achieve the skills that Trainee teachers must acquire during their formative process.

Keywords: tutored learning projects; formative evaluation; competency

\section{Introducción}

A partir del proceso de convergencia hacía el EEES en nuestro país se está produciendo un avance notable en el desarrollo de metodologías activas en la formación inicial del profesorado. Asimismo, el sistema ECTS ha supuesto un fuerte cambio en varios niveles:

1. Estructurar todo el sistema lectivo en torno a los procesos de aprendizaje del alumnado (Jarauta, 2014).

2. Buscar el desarrollo de una serie de competencias profesionales.

3. Desarrollar procesos de aprendizaje dialógico.

4. Utilizar procesos de evaluación formativa.

Un aspecto clave de estos nuevos paradigmas de aprendizaje es que están fuertemente relacionados con el desarrollo de la autonomía profesional (Araya \& España, 2012; Hortigüela, Salicetti, Hernando \& Pérez-Pueyo, 2015). La experiencia docente nos hace proponer los Proyectos de Aprendizaje Tutorado (PAT) como una estrategia muy válida para conseguir estos objetivos. Estos PAT se conciben como una oferta docente diseñada para promover el aprendizaje autónomo de los estudiantes, bajo la tutela del profesorado y cuya meta es la realización y evaluación de una tarea específica, un proyecto (Gil-Flores, Álvarez-Rojo, García-Jiménez \& RomeroRodríguez, 2005; Gómez, Gallego, Ibarra \& Rodríguez, 2010). 
Por tanto, el objetivo principal que se quiere alcanzar con este tipo de trabajos colaborativos es fomentar y desarrollar una variedad de competencias relacionadas no sólo con el trabajo en equipo, sino además potenciar la construcción del conocimiento del alumno como futuro profesional de la enseñanza, mediante el acompañamiento y supervisión del docente..

\section{Contextualización}

La experiencia de aplicación de los PAT se ha puesto en práctica en la asignatura optativa de 6 créditos del itinerario de Educación Física de $4^{\circ}$ del Grado de Educación Primaria durante el primer cuatrimestre en la Facultad de Educación de Segovia. Han participado 17 alumnos y la docencia se ha realizado en un pabellón deportivo y en las aulas anejas que éste posee.

En cuanto a las competencias profesionales que deben adquirir los estudiantes con estos PAT podemos destacar, entre las generales: Que los estudiantes sepan aplicar sus conocimientos y posean las competencias dentro de su área de estudio (la Educación). Y entre las específicas: Saber transformar el conocimiento y la comprensión de la Educación Física en procesos de enseñanza y aprendizaje adecuados.

\section{Diseño y desarrollo}

Carácter de la práctica: Llevar a cabo un proyecto de aprendizaje tutorado grupal sobre una temática propuesta. Grupos de 3-4 personas. El primer día de clase se hace el sorteo de los temas sobre los que hacer el PAT. Algunos temas propuestos: a) Cuentos y Canciones motrices; b) Retos y juegos cooperativos; c) Juegos populares e iniciación deportiva; entre otros.

- Estructura que debe tener cada documento a entregar antes de la puesta en práctica de la sesión programada

\section{A- Marco teórico del PAT:}

Introducción + análisis de temas más importantes de los documentos leídos + explicación de los recursos metodológicos empleados + capacidades físicas trabajadas + + redacción coherente e inteligible. Extensión máxima de 5 hojas. Después de la puesta en práctica, y antes de la evaluación de la sesión, los componentes del grupo deben explicar al resto de compañeros el marco teórico. 


\section{B- Plan de sesión}

1-Eje (objetivo principal de la sesión); 2-Contenido principal y secundarios; 3Metodología; 4-Actividades a realizar; 5-Agrupamientos y materiales; 6-Momentos de reflexión-acción; 7-Atención a la diversidad; 8-; 9-Roles de los profesores/as.

- Estructura que debe tener el informe final a realizar

-Datos y título en hoja inicial: "Informe del PAT..."

A- Evaluación del plan

Eje; contenido principal; contenidos secundarios (justificar cuándo se desarrollan), metodología (directiva / no directiva, tipo de tareas y estilos de enseñanza); evaluación (técnicas e instrumentos); atención a la diversidad (diversidad natural del grupo-aula y alumnos con NEE) y roles de los profesores.

B- Narrado (en tres columnas)

1- Fina: con el tiempo de la sesión en hora y minuto, al principio de cada actividad nueva.

2a - Ancha: Narrado detallado de lo que hace el grupo de alumnos y alumnas. “¿Qué veo?”. Descripción.

3a - Ancha: “¿Qué siento o interpreto acerca de lo que hace la persona observada y el grupo?

C- Análisis de las competencias docentes

Una vez realizada la puesta en práctica de la sesión, los compañeros valorarán las siguientes competencias docentes: 1- Práctica apta para todos; 2- Práctica gratificante; 3- Cada alumno es protagonista de su aprendizaje; 4- Se facilita el éxito; 5- Se implica a todos los alumnos; 6- Se favorece la participación; 7- Se recompensa el esfuerzo; 8- Se ofrece conocimiento; 9- Se secuencian correctamente las actividades; 10- Se realiza una evaluación.

\section{D- Evaluación del PAT}

Después de la puesta en práctica con los compañeros y con los alumnos de un colegio, donde se realizará una de las sesiones aplicadas en clase, se indicarán: a) variaciones 
respecto a lo planificado; y b) revisión del proceso seguido, problemas, soluciones, aprendizaje, valoración general, etc.).

\section{Evaluación}

Una vez acabado el cuatrimestre, se pasa un cuestionario anónimo para la valorar la experiencia. En una escala del 1 (nada) al 5 (mucho) deben emitir su juicio sobre los ítems planteados. Los resultados más destacados del cuestionario son los siguientes:

- $\quad$ Consideran útil lo aprendido con el PAT (4,88 sobre 5).

- La experiencia del trabajo de los PAT ha ayudado a adquirir competencias profesionales $(4,75)$

- $\quad$ La evaluación planteada favorece la adquisición de competencias profesionales $(4,69)$.

- $\quad$ Demuestra un impacto positivo y tangible de mejora $(4,56)$.

\section{Conclusiones}

Principales ventajas encontradas en la aplicación de los PAT y en la adquisición de competencias

- Genera una mayor autonomía en el aprendizaje.

- Mejoran sus estrategias de búsqueda de la información.

- Programan mejor.

- Elaboran su propio sistema de evaluación.

Problemas encontrados y posibles soluciones

- Elegir contenidos y temas más apropiados a los contenidos propios de la asignatura. Proponer a los alumnos que elijan el tema.

- Dificultades para reunirse el grupo. Plantear más momentos de encuentros grupales durante las clases teóricas y prácticas.

\section{Referencias}

Araya Muñoz, I., \& España-Chavarría, C. (2012). La autonomía profesional. Competencia clave para el docente en Educación Comercial, Revista Electrónica Educare, 16(2), 163-170. 
Gil-Flores, J., Álvarez-Rojo, V., García-Jiménez, E., \& Romero-Rodríguez, S. (2004). La Enseñanza Universitaria. Planificación y Desarrollo de la Docencia. Madrid: EOS Universitaria.

Gómez, M. Á., Gallego, B., Ibarra, M. S., \& Rodríguez, G. (2010). DiagWeb: Una experiencia de enseñanza basada en proyectos tutorados en Educación Superior mediante una WebQuest alojada en Moodle. Revista de Educación a Distancia, (24), 1-22.

Hortigüela, D., Salicetti, A., Hernando, A., \& Pérez-Pueyo, Á. (2015). El trabajo autónomo del alumno universitario en las clases de Educación Física. Análisis de su percepción sobre la práctica. Ágora para la Educación Física y el Deporte, 17(3), 251-265.

Jarauta Borrasca, B. (2014). El aprendizaje colaborativo en la universidad: Referentes y práctica. REDU. Revista de Docencia Universitaria, 12(4), 281-. 